Document downloaded from:

http://hdl.handle.net/10251/160442

This paper must be cited as:

Alegre Gil, MC.; Karapinar, E.; Marín Molina, J.; Tirado Peláez, P. (2019). Revisiting Bianchini and Grandolfi Theorem in the Context of Modified omega-Distances. Results in Mathematics. 74(4):1-9. https://doi.org/10.1007/s00025-019-1074-z

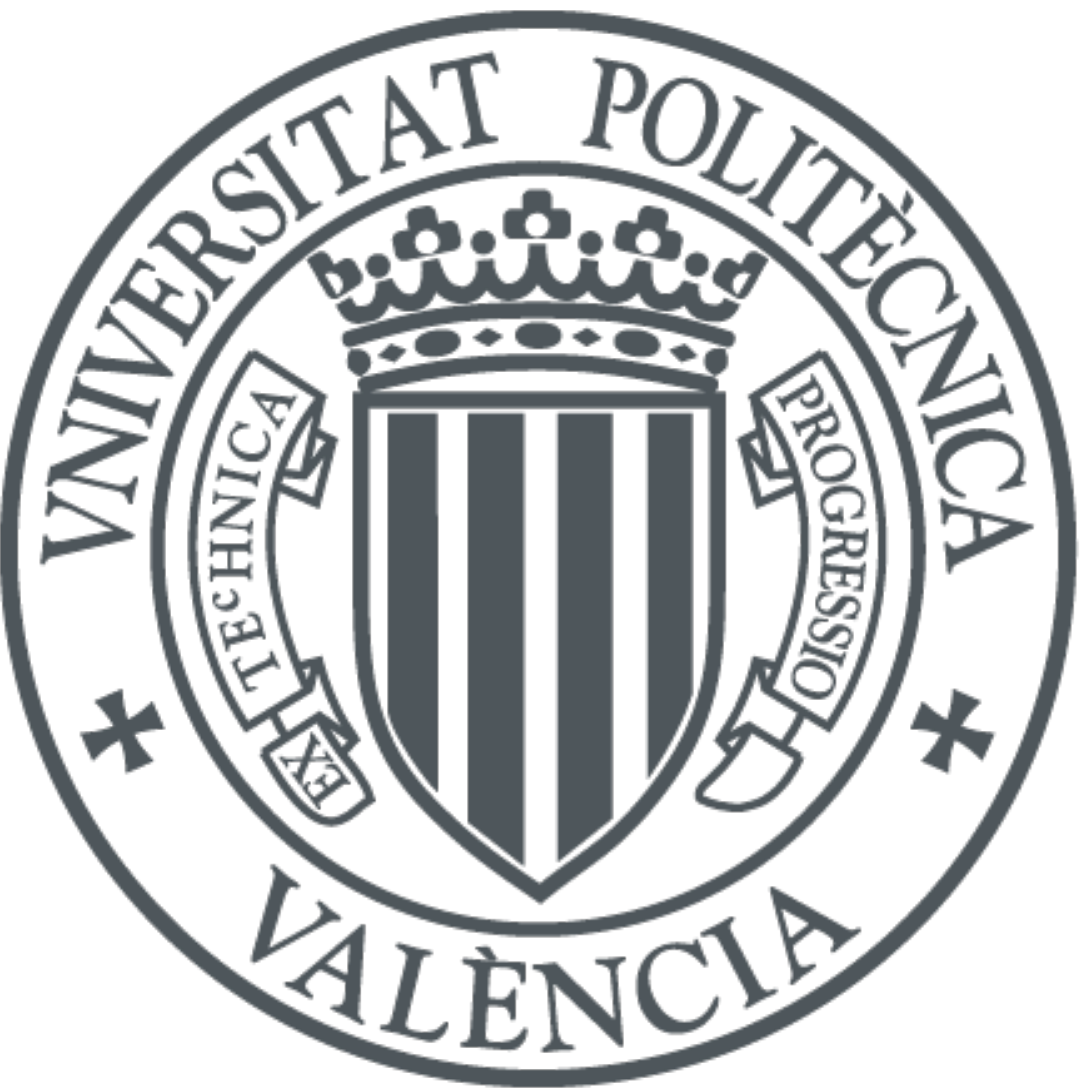

The final publication is available at

https://doi.org/10.1007/s00025-019-1074-z

Copyright Springer-Verlag

Additional Information 


\title{
Revisiting Bianchini and Grandolfi Theorem in the context of modified $\omega$-distances
}

\author{
Carmen Alegre Gil, Erdal Karapınar, Josefa Marín Molina, Pedro Tirado Peláez
}

\begin{abstract}
In this paper, we establish a proof for Bianchini and Grandolfi Theorem in the context of quasi-metric spaces via modified $\omega$-distances. As consequences of our main results, we derive several existing fixed point theorems in the literature. Various examples are presented to illustrate our obtained results.
\end{abstract}

\section{Introduction}

In [6] Kada et al. proposed the notion of $\omega$-distance over a metric and they proved a fixed point theorem in which the contraction condition was given by the $\omega$-distance instead of the metric. As it is expected, following this initial paper, the analogous of several renowned fixed point theorems have been proved in this new framework. Later on, Park in [14] extended the notion of $\omega$-distance to quasi-metric spaces and this concept has been used in some directions in order to obtain fixed point results on complete quasi-metric spaces (see e.g. $[\mathbf{1}],[\mathbf{3}],[\mathbf{1 1}],[\mathbf{1 3}]$ and the references therein). If $d$ is a metric on $X$, then $d$ is a $\omega$-distance on the metric space $(X, d)$. Nevertheless, if $d$ is a quasimetric on $X$, then $d$ is not necessarily a $\omega$-distance on the quasi-metric space $(X, d)$. Recently, in 2016, C. Alegre and J. Marín [2] solved this problem by introducing the notion of mw-distance (modified $\omega$-distance).

For the sake of completeness, we state the definition of $m \omega$-distance here.

Definition 1. (Definition 3 of [2]) An mw-distance on a quasi-metric space $(X, d)$ is a function $q: X \times X \rightarrow \mathbb{R}_{0}^{+}$satisfying the following conditions:

$(m \omega 1) q(x, y) \leq q(x, z)+q(z, y)$ for all $x, y, z \in X$;

$(m \omega 2) q(x, \cdot): X \rightarrow \mathbb{R}_{0}^{+}$is lower semicontinuous on $\left(X, \tau_{d^{-1}}\right)$ for all $x \in X$;

(mw3) for each $\varepsilon>0$ there exists $\delta>0$ such that if $q(y, x) \leq \delta$ and $q(x, z) \leq \delta$ then $d(y, z) \leq \varepsilon$.

We underline the fact that every quasi-metric $d$ on $X$ is an mw-distance on the quasi-metric space $(X, d)$.

EXAMPLE 2. (Example 7 of $[\mathbf{2}])$. Let $(X, p)$ be an asymmetric normed space. Let $d_{p}$ the quasi-metric induced by $p$, namely $d_{p}(x, y)=p(y-x)$. Then $q: X \times X \rightarrow \mathbb{R}_{0}^{+}$defined by $q(x, y)=p(-x)+p(y)$ is an mw-distance on the quasi-metric space $\left(X, d_{p}\right)$.

For more examples and details on $m \omega$-distances, we refer [2].

2000 Mathematics Subject Classification. 47H10; 54H25.

Key words and phrases. $\omega$-distance, modified- $\omega$-distance, quasi-metric space, Bianchini and Grandolfi function, (C)-comparison function, fixed point. 
Definition 3. (Definition 4 of $[2]$ ) A strong mw-distance on a quasi-metric space $(X, d)$ is a mw-distance $q: X \times X \rightarrow \mathbb{R}_{0}^{+}$satisfying the following condition:

$\left(m \omega 2^{\prime}\right) q(\cdot, x): X \rightarrow \mathbb{R}_{0}^{+}$is lower semicontinuous on $\left(X, \tau_{d^{-1}}\right)$ for all $x \in X$.

We also need the following notion.

DeFinition 4. Let $q$ be an mw-distance on a quasi-metric space $(X, d)$. We say that a map $f: X \rightarrow X$ is q-lower semicontinuous (q-l.s.c in short) if the function $x \rightarrow q(x, f x)$ is lower semicontinuous on the metric space $\left(X, d^{s}\right)$.

In 1968, Bianchini and Grandolfi proposed an interesting extension of the renowned Banach contraction principle as follows.

Theorem.(Bianchini and Grandolfi $[4]$ ) Let $f$ be a self mapping of a complete metric space $(X, d)$ such that for each $x, y \in X$

$$
d(f x, f y) \leq \varphi(d(x, y))
$$

where $\varphi:[0, \infty) \rightarrow[0, \infty)$ is a nondecreasing function satisfying $\sum_{n=0}^{\infty} \varphi^{n}(t)<\infty$ for all $t>0$. Then $f$ has a unique fixed point.

A function $\varphi$ satisfying the conditions of this theorem is called a Bianchini-Grandolfi gauge function in some sources, e.g. [15], and is also known as $(c)$-comparison functions ([16]). Notice that if $\varphi$ is a Bianchini-Grandfolfi gauge function, then $\varphi(t)<t$ for any $t>0$ (see e.g. [16]).

Recently this theorem has been generalized in different ways, see e.g. $[\mathbf{8}, \mathbf{9}]$. In particular, in $[\mathbf{7}$, 12] the authors extend the Bianchini-Grandfolfi theorem to the framework of complete quasi-metric spaces replacing in the contraction condition the quasi-metric by a $Q$-function. On the other hand, in [2], the authors introduced the notion of $m \omega$-distance on a quasi-metric space which generalizes the concept of quasi-metric and they obtained a fixed point theorem for generalized contractions with respect to $m \omega$-distances and Jachymski functions on complete quasi-metric spaces which also generalizes the Bianchini and Grandolfi theorem. In this paper we obtain several fixed point results for self maps defined on complete quasi-metric spaces satisfying a contraction condition in terms of $m \omega$-distances and using Bianchini-Grandolfi gauge functions.

We start by recalling several notions and properties of the theory of quasi-metric spaces. Our basic references are $[5, \mathbf{1 0}]$.

A quasi-metric on a set $X$ is a function $d: X \times X \rightarrow \mathbb{R}_{0}^{+}$such that for all $x, y, z \in X:$ (i) $d(x, y)=d(y, x)=0$ if and only if $x=y$ (ii) $d(x, y) \leq d(x, z)+d(z, y)$.

Each quasi-metric $d$ on a set $X$ induces a $T_{0}$ topology $\tau_{d}$ on $X$ which has as a base the family of open balls $\left\{B_{d}(x, \varepsilon): x \in X, \varepsilon>0\right\}$, where $B_{d}(x, \varepsilon)=\{y \in X: d(x, y)<\varepsilon\}$ for all $x \in X$ and $\varepsilon>0$.

Given a quasi-metric $d$ on $X$, the function $d^{-1}$ defined by $d^{-1}(x, y)=d(y, x)$ for all $x, y \in X$, is also a quasi-metric on $X$, called conjugate quasi-metric, and the function $d^{s}$ defined by $d^{s}(x, y)=$ $\max \{d(x, y), d(y, x)\}$ for all $x, y \in X$, is a metric on $X$.

A quasi-metric space $(X, d)$ is called complete if every Cauchy sequence $\left\{x_{n}\right\}_{n \in \omega}$ in the metric space $\left(X, d^{s}\right)$ converges with respect to the topology $\tau_{d^{-1}}$ (i.e., there exists $z \in X$ such that $\left.d\left(x_{n}, z\right) \rightarrow 0\right)$. Throughout this paper the letter $\omega$ will denote the set of non-negative integer numbers.

\section{Fixed point theorems}

TheOREM 5. Let $(X, d)$ be a complete quasi-metric space, $q$ a strong mw-distance on $(X, d)$, $\varphi:[0, \infty) \rightarrow[0, \infty)$ a Bianchini-Grandolfi gauge function and $f: X \rightarrow X$ a q-l.s.c map satisfying 


$$
\begin{aligned}
& q(f x, f y) \leq \varphi(\max \{q(x, y), q(x, f x), q(y, f y)\}) \\
& q(f x, f y) \leq \varphi(\max \{q(x, y), q(f x, x), q(f y, y)\})
\end{aligned}
$$

for all $x, y \in X$, then $f$ has a unique fixed point $z \in X$.

Proof. Fix $x_{0} \in X$ and let $x_{n}=f^{n} x$ for each $n \in \mathbb{N}$. By using a similar technique to the one given in [7] we shall prove that $\left\{x_{n}\right\}_{n \in \omega}$ is a Cauchy sequence in $\left(X, d^{s}\right)$. Indeed, by (1) we have

$$
q\left(x_{n+1}, x_{n+2}\right) \leq \varphi\left(\max \left\{q\left(x_{n}, x_{n+1}\right), q\left(x_{n+1}, x_{n+2}\right)\right\}\right)
$$

for all $n \in \omega$.

Now we distinguish two cases.

Case 1. There exists $k \in \omega$ such that $q\left(x_{k}, x_{k+1}\right)=0$. Then, by condition (3) and the fact that $\varphi(t)<t$ for all $t>0$, we deduce that $q\left(x_{k+1}, x_{k+2}\right)=0$. Repeating this argument, we obtain $q\left(x_{k+j}, x_{k+j+1}\right)=0$ for all $j \in \omega$. So, by condition $(m \omega 1), q\left(x_{n}, x_{m}\right)=0$ whenever $m>n \geq k$.

Case 2. $q\left(x_{n}, x_{n+1}\right)>0$ for all $n \in \omega$. Then, by (3) and the fact that $\varphi(t)<t$ for all $t>0$, we deduce that $q\left(x_{n}, x_{n+1}\right)>q\left(x_{n+1}, x_{n+2}\right)$ for all $n \in \omega$, so

$$
q\left(x_{n+1}, x_{n+2}\right) \leq \varphi\left(q\left(x_{n}, x_{n+1}\right)\right)<q\left(x_{n}, x_{n+1}\right),
$$

for all $n \in \omega$. Therefore

$$
q\left(x_{n}, x_{n+1}\right) \leq \varphi^{n}\left(q\left(x_{0}, x_{1}\right)\right)
$$

for all $n \in \omega$. Now choose and arbitrary $\varepsilon>0$ and let $\delta=\delta(\varepsilon) \in(0, \varepsilon)$ for which condition $(m \omega 3)$ holds. Since $q\left(x_{0}, x_{1}\right)>0$ and $\sum_{n=0}^{\infty} \varphi^{n}\left(q\left(x_{0}, x_{1}\right)\right)<\infty$, there is $n_{\delta} \in \omega$ such that $\sum_{n=n_{\delta}}^{\infty} \varphi^{n}\left(q\left(x_{0}, x_{1}\right)\right)<\delta$. Then, for $m>n \geq n_{\delta}$, we obtain, by $(m \omega 1)$,

$$
\begin{aligned}
q\left(x_{n}, x_{m}\right) & \leq q\left(x_{n}, x_{n+1}\right)+q\left(x_{n+1}, x_{n+2}\right)+\ldots+q\left(x_{m-1}, x_{m}\right) \\
& \leq \varphi^{n}\left(q\left(x_{0}, x_{1}\right)\right)+\varphi^{n+1}\left(q\left(x_{0}, x_{1}\right)\right)+\ldots+\varphi^{m-1}\left(q\left(x_{0}, x_{1}\right)\right) \\
& \leq \sum_{j=n_{\delta}}^{\infty} \varphi^{j}\left(q\left(x_{0}, x_{1}\right)\right)<\delta .
\end{aligned}
$$

Then, $q\left(x_{n}, x_{m}\right)<\delta$ for all $m>n \geq n_{\delta}$. Similarly, by (2) we have

$$
q\left(x_{n+2}, x_{n+1}\right) \leq \varphi\left(\max \left\{q\left(x_{n+1}, x_{n}\right), q\left(x_{n+2}, x_{n+1}\right)\right\}\right),
$$

for all $n \in \omega$. By processing as in the previous case we can show that there exists $n_{\delta}^{\prime}$ such that $q\left(x_{m}, x_{n}\right)<\delta$ for all $m>n \geq n_{\delta}^{\prime}$.

Now let $i, j \in \mathbb{N}$ with $j>i>k_{\delta}=\max \left\{n_{\delta}, n_{\delta}^{\prime}\right\}$. Hence by $(m \omega 3)$ we have $q\left(x_{k_{\delta}}, x_{j}\right)<\delta$ and $q\left(x_{i}, x_{k_{\delta}}\right)<\delta$ so $d\left(x_{i}, x_{j}\right) \leq \varepsilon$. Similarly $q\left(x_{k_{\delta}}, x_{i}\right)<\delta$ and $q\left(x_{j}, x_{k_{\delta}}\right)<\delta$ so $d\left(x_{j}, x_{i}\right) \leq \varepsilon$. Therefore $\left\{x_{n}\right\}$ is a Cauchy sequence in $\left(X, d^{s}\right)$. Since $(X, d)$ is complete, there exists $z \in X$ such 
that $d\left(x_{n}, z\right) \rightarrow 0$.

Now we shall prove that $q\left(x_{n}, z\right) \rightarrow 0$ and $q\left(z, x_{n}\right) \rightarrow 0$. Indeed, chosen an arbitrary $\varepsilon>0$, since $d\left(x_{n}, z\right) \rightarrow 0$ it follows from $(m \omega 2)$ that for $m, n$ sufficiently large $q\left(x_{n}, z\right)<q\left(x_{n}, x_{m}\right)+\varepsilon$, then $q\left(x_{n}, z\right) \rightarrow 0$. Similarly, by $\left(m \omega 2^{\prime}\right), q\left(z, x_{n}\right)<q\left(x_{m}, x_{n}\right)+\varepsilon$, so $q\left(z, x_{n}\right) \rightarrow 0$. Therefore, by $(m \omega 1), q(z, z)=0$.

Next we show that $d\left(z, x_{n}\right) \rightarrow 0$. Indeed, given $\varepsilon>0$ take $\delta=\delta(\varepsilon) \in(0, \varepsilon)$ for which condition (mw3) holds. Since $q\left(z, x_{m}\right)<\delta$ and $q\left(x_{m}, x_{n}\right)<\delta$ for $m, n$ sufficiently large, it follows that $d\left(z, x_{n}\right)<\varepsilon$. Taking into account that $f$ is $q-l s c$ we deduce that $q(z, f z)=0$.

Since by (1)

$$
q\left(x_{n+1}, f z\right) \leq \varphi\left(\max \left\{q\left(x_{n}, z\right), q\left(x_{n}, x_{n+1}\right), q(z, f z)\right\}\right)
$$

we have that $q\left(x_{n}, f z\right) \rightarrow 0$.

Since $q\left(x_{n}, z\right) \rightarrow 0$ and $q(z, f z)=0$, by $(m \omega 3)$, we have that $d\left(x_{n}, f z\right) \rightarrow 0$. Then, by $\left(m \omega 2^{\prime}\right)$, we have that given $\varepsilon>0$ there exits $n_{\varepsilon}$ such that $q(f z, z)<q\left(x_{n}, z\right)+\varepsilon$ for all $n \geq n_{\varepsilon}$, therefore $q(f z, z)=0$. So, by $(m \omega 1)$, we have that $q(f z, f z)=0$ and consequently, by $(m \omega 3)$, $d(z, f z)=d(f z, z)=0$. We conclude that $z=f z$.

Finally, let $u \in X$ such that $f u=u$, then by (1) or (2) $q(u, u)=q(f u, f u)<q(u, u)$, so $q(u, u)=0$. Similarly $q(u, z)=q(f u, f z)<q(u, z)$, so $q(u, z)=0$ and by $(m \omega 3), d(u, z)=$ $d(z, u)=0$. Consequently $u=z$, and $z$ is the unique fixed point.

As the following theorem shows, if contraction conditions (1) and (2) are changed, the $q$-lower semicontinuity of $f$ can be removed.

THEOREM 6. Let $(X, d)$ be a complete quasi-metric space, $q$ a strong mw-distance on $(X, d), \varphi$ : $[0, \infty) \rightarrow[0, \infty)$ a Bianchini-Grandolfi gauge function and $f: X \rightarrow X$ a self-map satisfying

$$
\begin{aligned}
& q(f x, f y) \leq \varphi(\max \{q(x, y), q(x, f x)\}) \\
& q(f x, f y) \leq \varphi(\max \{q(x, y), q(f x, x)\})
\end{aligned}
$$

for all $x, y \in X$, then $f$ has a unique fixed point $z \in X$.

Proof. Fix $x_{0} \in X$ and let $x_{n}=f^{n} x_{0}$ for each $n \in \mathbb{N}$. By using the same proof that in the previous theorem we obtain $z \in X$ such that $q\left(z, x_{n}\right) \rightarrow 0, q\left(x_{n}, z\right) \rightarrow 0$ and $q(z, z)=0$.

By condition (5), we have that

$$
q\left(x_{n}, f z\right) \leq \varphi\left(\max \left\{q\left(x_{n-1}, z\right), q\left(x_{n-1}, x_{n}\right)\right\}\right),
$$

for all $n \in \mathbb{N}$. Therefore, $q\left(x_{n}, f z\right) \rightarrow 0$.

Since $q(z, f z) \leq q\left(z, x_{n}\right)+q\left(x_{n}, f z\right)$, we obtain that $q(z, f z)=0$.

Now, by condition (6), we have that

$$
q\left(f z, x_{n}\right) \leq \varphi\left(\max \left\{q\left(z, x_{n-1}\right), q(z, f z)\right\}\right),
$$

for all $n \in \mathbb{N}$. Therefore, $q\left(f z, x_{n}\right) \rightarrow 0$.

Finally, since $q\left(f z, x_{n}\right) \rightarrow 0$ and $q\left(x_{n}, z\right) \rightarrow 0$, by $(m \omega 3)$ we have that $d(f z, z)=0$.

On the other hand, since $q\left(z, x_{n}\right) \rightarrow 0$ and $q\left(x_{n}, f z\right) \rightarrow 0$, by $(m \omega 3)$ we have that $d(f z, z)=0$. 
Consequently, $z=f z$.

The proof of the uniqueness of the fixed point is as in above theorem.

\section{Consequences}

The following immediate consequence is obtained by choosing Bianchini-Grandolfi gauge function $\varphi(t)=k t$ where $k \in[0,1)$ in Theorem 5 .

Corollary 7. Let $(X, d)$ be a complete quasi-metric space, $q$ a strong mw-distance on $(X, d)$, and $f: X \rightarrow X$ a q-l.s.c map. If there exists $k \in[0,1)$ such that

$$
\begin{aligned}
& q(f x, f y) \leq k \max \{q(x, y), q(x, f x), q(y, f y)\} \\
& q(f x, f y) \leq k \max \{q(x, y), q(f x, x), q(f y, y)\}
\end{aligned}
$$

for all $x, y \in X$, then $f$ has a unique fixed point $z \in X$.

The following immediate consequence is obtained by choosing Bianchini-Grandolfi gauge function $\varphi(t)=k t$ where $k \in[0,1)$ in Theorem 6 .

Corollary 8. Let $(X, d)$ be a complete quasi-metric space, q a strong mw-distance on $(X, d)$, and $f: X \rightarrow X$ a self-map. If there exists $k \in[0,1)$ such that

$$
\begin{aligned}
& q(f x, f y) \leq k(\max \{q(x, y), q(x, f x)\}) \\
& q(f x, f y) \leq k(\max \{q(x, y), q(f x, x)\})
\end{aligned}
$$

for all $x, y \in X$, then $f$ has a unique fixed point $z \in X$.

The next corollary is a version in terms of $m \omega$-distances of the theorem obtained in [7] for $Q$-functions.

Corollary 9. Let $(X, d)$ be a complete quasi-metric space, $q$ a strong mw-distance on $(X, d), \varphi$ : $[0, \infty) \rightarrow[0, \infty)$ a Bianchini-Grandolfi gauge function and $f: X \rightarrow X$ a self-map satisfying

$$
q(f x, f y) \leq \varphi(q(x, y))
$$

for all $x, y \in X$, then $f$ has a unique fixed point $z \in X$.

The following immediate consequence is obtained by choosing Bianchini-Grandolfi gauge function $\varphi(t)=k t$ where $k \in[0,1)$ in Corollary 9 .

Corollary 10. Let $(X, d)$ be a complete quasi-metric space, $q$ a strong mw-distance on $(X, d)$, and $f: X \rightarrow X$ a self-map. If there exists a $k \in[0,1)$ such that

$$
q(f x, f y) \leq k(q(x, y))
$$

for all $x, y \in X$, then $f$ has a unique fixed point $z \in X$. 
The following example shows that in Theorem 5, Theorem 6 and Corollary 9 the strong condition for the $m \omega$-distance cannot be omitted.

Example 1. ( See Example 10 of [2]). Let $X=\{1 / n: n \in \mathbb{N}\}$ and let $d$ be the quasi-metric on $X$ given by $d(x, x)=0$, and $d(x, y)=x .(X, d)$ is a complete quasi-metric space.

The function $q(x, y)=d(x, y)$ is an $m \omega$-distance and it is not strong.

Let $f: X \rightarrow X$ given by $f x=x / 3$ and let $\phi: \mathbb{R}_{0}^{+} \rightarrow \mathbb{R}_{0}^{+}$given by $\phi(t)=t / 2$. Then $\phi$ is a Bianchini-Grandolfi gauge function and it satisfies the conditions (1) and (2) because $\phi(q(x, y))=$ $\phi(x)=\frac{x}{2}>\frac{x}{3}=f x=q(f x, f y)$, for all $x, y \in X$.

$f$ is a $q$-lower semicontinuous map because if $d^{s}\left(x_{n}, x\right) \rightarrow 0$, then there exists $n_{0}$ such that $x_{n}=x$ for all $n \geq n_{0}$, then $q(x, f x)-q\left(x_{n}, f x_{n}\right)=0$ for all $n \geq n_{0}$.

Nevertheless, $f$ has not fixed point in $X$.

Next, we give an example where we can apply Theorem 6 and Corollary 9 but not Theorem 5 .

Example 2. Let $X=\mathbb{R}_{0}^{+}$and let $d$ be the quasi-metric given by $\left.d(x, y)=\max \{y-x, 0)\right\}$. Let $q$ be the $m \omega$-distance given $q(x, y)=y .(X, d)$ is a complete quasi-metric space and $q$ is a strong $m \omega$-distance on $(X, d)$ (see Examples 5 and 11 of $[2]$ ).

Let $f: X \rightarrow X$ given by $f x=x / 2$ if $x \in[0,2)$ and $f x=n$ if $x \in[n+1, n+2)$ where $n \in \mathbb{N}$.

Let $\phi=f$. Then $\phi$ is a Bianchini-Grandolfi gauge function and it satisfies the conditions of Theorem 6 and Corollary 9 because $\phi(q(x, y))=\phi(y)=f y=q(f x, f y)$, for all $x, y \in X$.

In fact, $z=0$ is the unique fixed point of $x$. Nevertheless, we can not apply Theorem 5 because $f$ is not $q$-lower semicontinuos. In effect, $d^{s}\left(3-\frac{1}{n}, 3\right) \rightarrow 0$ and $q(3, f 3)-q\left(3-\frac{1}{n}, f\left(3-\frac{1}{n}\right)\right)=1$.

Remark 1. As mentioned in Introduction, in [2] the authors obtained a fixed point theorem for generalized contractions with respect to $m \omega$-distances and Jachymski functions on complete quasi-metric spaces which also generalizes the Bianchini and Grandolfi theorem. Let us recall that a function $\phi:[0, \infty) \rightarrow[0, \infty)$ is said to be a Jachymski function if $\phi(0)=0$ and for each $\varepsilon>0$ there exists $\delta>0$ such that for $t>0$ with $\varepsilon<t<\varepsilon+\delta$, we have $\phi(t) \leq \varepsilon$.

If $\varphi:[0, \infty) \rightarrow[0, \infty)$ a Bianchini-Grandolfi gauge function then it is a Jachymski function. In effect, if $\varphi$ is not Jachymski, there exists $\varepsilon>0$ such that for all $n \in \mathbb{N}$ there is $t_{n} \in(\varepsilon, \varepsilon+1 / n)$ such that $\varphi\left(t_{n}\right)>\varepsilon$. If $t>\varepsilon$, there exists $n_{0}$ such $t>t_{n_{0}}$. Since $\varphi$ is nondeacreasing, $\varphi(t)>\varepsilon$. Applying the same argument to $\varphi(t)>\varepsilon$ we have that $\varphi^{2}(t)>\varepsilon$. Following this reasoning, we obtain that $\varphi^{n}(t)>\varepsilon$, for all $n \in \mathbb{N}$. Consequently, $\left.\sum_{n=1}^{\infty} \varphi^{n} t\right)=+\infty$ and this is a contradiction.

Therefore, Corollary 9 can also be obtained from Theorem 1 of [2].

Remark 2. The class of Bianchini-Grandolfi functions is strictly contained in the class of Jachymski functions. The function $\varphi(t)=0$, for all $t \neq 1$ and $\varphi(1)=1$ is Jachymski and it is not Bianchini-Grandolfi, because if it is then $\varphi(t)<t$, for all $t>0$.

If the Jachymski function satisfies that $\varphi(t)<t$, for all $t>0$, the function does not be Bianchini-Grandolfi. For instance, the Jachymski function $\varphi(t)=t / 2$ if $t \in(1,2]$ and $\varphi(t)=0$ otherwise, satisfies the above condition and it is not Bianchini-Grandolfi because this function is not nondecreasing,

The natural question that arises is to establish whether Theorem 5 and Theorem 6 remain true for Jachymski functions.

\section{References}

[1] C. Alegre, J. Marín and S. Romaguera, A fixed point theorem for generalized contractions involving w-distances on complete quasi-metric spaces, Fixed Point Theory and Applications, 2014, 2014:40

[2] C. Alegre and J. Marín, Modified w-distances on quasi-metric spaces and a fixed point theorem on complete quasi-metric spaces, Topology and its Applications 203 (2016) 32-41. 
[3] S. Al-Homidan, Q.H. Ansari, J.C. Yao, Some generalizations of Ekeland-type variational principle with applications to equilibrium problems and fixed point theory, Nonlinear Analysis: Theory, Methods \& Applications 69 (2008) 126-139.

[4] R.M. Bianchini and M. Grandolfi, Trasformazioni di tipo contrattivo generalizatto in uno spacio metrico, Atti della Accademia Nazionale dei Lincei 45 (1969) 212-216.

[5] S. Cobzas, Functional Analysis in Asymmetric Normed Spaces, Birkhauser, Basel, 2013.

[6] O. Kada, T. Suzuki, W.Takahashi, Nonconvex minimization theorems and fixed point theorems in complete metric spaces, Mathematica Japonica, 44 (1996) 381-391.

[7] E. Karapinar, S. Romaguera and P. Tirado, Contractive multivalued maps in terms of Q-functions on complete quasimetric spaces, Fixed Point Theory and Applications 2014, 2014:53.

[8] H.Alsulami, S. Gulyaz, E. Karapinar and I.M. Erhan, Fixed point theorems for a class of $\alpha$-admissible contractions and applications to boundary value problem, Abstract and Applied Analysis, (2014) Article Id: 187031

[9] E. Karapinar, B. Samet, Generalized $(\alpha-\psi)$ contractive type mappings and related fixed point theorems with applications, Abstr. Appl. Anal , 2012 (2012) Article id: 793486

[10] H.P.A. Künzi, Nonsymmetric distances and their associated topologies: About the origins of basic ideas in the area of asymmetric topology, in: C.E. Aull, R. Lowen (Eds.), Handbook of the History of General Topology, 3, Kluwer, Dordrecht (2001) 853-968.

[11] J. Marín, S. Romaguera, P. Tirado, Generalized Contractive Set-Valued Maps on Complete Preordered QuasiMetric Spaces, Journal of Functions Spaces and Applications, Article ID 269246 (2013), 6 pages.

[12] J.Marín, S. Romaguera and P. Tirado, Q-Functons on Quasimetric spaces and fixed points for multivalued maps. Fixed Point Theory and Appllications, 2011, Article ID603861 (2011)

[13] J. Marín, S. Romaguera, P. Tirado, Weakly contractive multivalued maps and w-distances on complete quasimetric spaces, Fixed Point Theory and Applications, 1 (2011) 1-9.

[14] S. Park, On generalizations of the Ekeland-type variational principles, Nonlinear Analysis: Theory, Methods \& Applications, 39 (2000) 881-889.

[15] P.D. Proinov. A generalization of the Banach contraction principle with high order of convergence of successive approximations Nonlinear Analysis, Theory, Methods and Applications 67 (2007) 2361-2369.

[16] I. A. Rus, Generalized contractions and applications, Cluj University Press, Cluj-Napoca, 2001.

Carmen Alegre Gil, instituto Universitario de Matemática Pura y Aplicada, Universitat Politècnica de València, Camí de Vera s/n, 46022 Valencia, Spain

Erdal Karapinar, Department of Medical Research, China Medical University Hospital, China Medical University, 40402, Taichung, TaiWan

E-mail address: erdalkarapinar@yahoo.com

Josefa Marín Molina, Instituto Universitario de Matemática Pura y Aplicada, Universitat Politècnica de València, Camí de Vera s/n, 46022 Valencia, Spain

Pedro Tirado Peláez, Instituto Universitario de Matemática Pura y Aplicada, Universitat Politècnica de València, Camí de Vera s/n, 46022 Valencia, Spain 